\title{
Status Analysis on the Marketing Authorization Holder of China's Drugs
}

\author{
Yu Haishi, Jiang Yingzhu and Zhou Weijun \\ Center for Certification and Evaluation, Guangdong Drug Administration, Guangzhou, 510080
}

\begin{abstract}
We analyze the basic situation of Marketing Authorization Holder (MAH) of drugs in China. Mathematical analysis was carried out on the approval time, product category, dosage form, listing permit holder and production unit of 3239 MAH of drugs in China as of July 31, 2019. We found the following results. The approval time for MAH of drugs was concentrated in 2015, mainly based on chemicals, and the dosage forms were mostly tablets and injections. Furthermore, the number of MAH of drugs varies greatly among different provinces, and the number of Hebei, Guangdong, and Jiangsu ranks in the top three. Thirdly, there are time differences, variety types and geographical differences in the MAH of drugs. This is the application for examination and approval after the full implementation of the MAH of drugs system, and the patent and intellectual property protection in the process of entrusted production technology transfer. The research provides reference for enterprise declaration and regulatory approval management after the full implementation of the MAH system in China.
\end{abstract}

\section{Introduction}

Marketing Authorization Holder (MAH) of drugs refers to entities such as drug R\&D institutions, scientific researchers, and drug manufacturers with drug technology, a system for obtaining a drug marketing authorization by submitting a drug marketing authorization application and taking primary responsibility for the quality of the drug throughout its life cycle. The system originated from European and American countries, and it is a system model that separates the drug marketing authorization from the production permission. It allows drug $\mathrm{MAH}$ produce medicines by themselves, or entrust other production enterprises to produce medicines. Drug MAH are holders of drug marketing authorization documents, including research and development institutions, scientific researchers, and drug manufacturing enterprises. [1-4]

In order to better meet the growing health needs of the people, and to explore and promote the reform and innovation of China's drug review and approval system, in August 2015, the State Council issued the Opinions on Reforming the System for the Review and Approval of Drug Therapy Devices (State Council [2015] No. 44), proposed to carry out MAH degree pilot, opened the curtain of deepening the reform of China's drug regulatory system.[5]In November 2015, the National People's Congress issued the "Decision of the Standing Committee of the National People's Congress on Authorizing the State Council to Carry out Pilots and Related Issues of the Drug Marketing Authorization Holder System in Certain Places", and explicitly authorized Beijing, Shanghai, Guangdong and other 10 provinces and cities to conduct pilots[6].

The State Council and the former State Food and Drug Administration also successively formulated corresponding documents. For example, in June 2016, the General Office of the State Council printed and issued the "Notice of the General Office of the State Council on Printing and Distributing Pilot Programs for Holders of Drug Marketing Authorization" (State Affairs Office [2016] No. 41) [7]. In August 2017, the former General Administration of Food and Drug Administration issued the "Notice of the General Administration on Promoting the Pilot Work of the Drug Marketing Authorization Holder System" (Food and Drug Administration and Pharmaceutical Management [2017] No. 68) [8] and so on. On October 23, 2017, the State Council issued the "Amendment to the Drug Administration Law of the People's Republic of China (Draft for Soliciting Drafts)", which intends to fully implement the drug marketing authorization holder system[9]. In order to better summarize the pilot experience of the drug marketing license holder system, lay a solid foundation for the reform and improvement of the drug management system, and connect the pilot work of the drug marketing license holder system with the revision of the Drug Administration Law of the People's Republic of China, The sixth meeting of the Standing Committee of the Thirteenth National People's Congress in October 2018 decided to authorize the State Council to conduct drug marketing license holding in some places at the seventeenth meeting of the Standing Committee of the Twelfth National People's Congress in November 2015 The three-year period for pilot work on the human system 
was extended by one year. In August 2019, the State Drug Administration's Drug Marketing Authorization Database was launched, and the holder database shared platform displayed related information of the drug MAH and their products that have been approved for marketing. This provides a data basis for comprehensively sorting out and analyzing the basic situation of the varieties of Chinese pharmaceutical MAH.

In view of this, this article records the types of MAH, main bodies of MAH, production units, approval time, dosage forms, product categories, etc. recorded in the database of Chinese drug MAH (as of July 31, 2019) The situation is analyzed by mathematical statistics. It is expected to provide references and references for the basic cognition and related research of Chinese drug marketing licensee holders by academia, regulatory authorities, and drug manufacturers.

\section{Materials and Methods}

\subsection{Definition of analysis objects}

The analysis included the varieties of holders of domestic drug marketing licenses (3239, based on the number of drug approval documents). Analyze the approval time, product category, dosage form, marketing authorization holder, production unit and geographical distribution of these varieties.

\subsection{Data Sources}

The Drug Marketing Authorization Database of the State Drug Administration was officially launched on August 2, 2019. This article screens out entries for MAH from the domestic drug entry information (165323 records) published on the official website of the State Drug Administration(http://www.nmpa.gov.cn/WS04/CL2042/ ), a total of 3239 article. These data are as of July 31, 2019, and the search query time is August 3, 2018August 7, 2018. Article 3239 The earliest approval date for the variety of MAH is March 1, 2014.

\subsection{Research methods}

\subsubsection{Statistical analysis and comparative analysis}

The paper uses Excel 2007 to sort and sort 3239 MAH' $^{\prime}$ variety data to form a basic database. Then sort the approval time, product category, dosage form, marketing authorization holder, and production unit, and perform statistics on quantity and proportion. On this basis, the varieties, MAH, and production units are spatially classified according to provinces. The above information is combed and analyzed in detail in order to provide a reference for the research and approval of drug MAH.

\subsubsection{Chi-square test}

The Chi-Square test analysis method is used to perform Chi-Square tests on the distribution of MAH varieties and all varieties. If the Chi-Square testing is passed, it indicates that there is a statistical basis for the differences in the MAH's variety system.

The inspection process is as follows:

(1) Put forward the null hypothesis:

H0: Overall sample $X$ is $F(x)$.

If the overall distribution is discrete, it is assumed that $\mathrm{H} 0$ : The distribution law of $\mathrm{X}$ is:

$$
P\left\{X=x_{i}\right\}=p_{i},(i=1,2, \ldots)
$$

(2) Divide the value range of $X$ into $k$ disjoint intervals $A_{1}, A_{2}, A_{3}, \ldots, A_{k}$, where as:

$$
A_{1}=\left(a_{0}, a_{1}\right], A_{2}=\left(a_{1}, a_{2}\right], \ldots, A_{k}=\left(a_{k-1}, a_{k}\right)
$$

Where $a_{0}$ can take $-\infty, a_{k}$ can take $+\infty$, the division of the interval depends on the specific situation, but the number of sample values contained in each small interval should not be less than 5 , and the number of intervals $k$ should not be too large or too small.

(3) Let $f_{i}$ be the number of sample values of $A_{i}$ that fall into the $i$-th interval, and become the group frequency (true value). The sum of all group frequencies $f_{1}+f_{2}+\ldots$ $+f_{k}$ is equal to the sample size $n$.

(4) When HO is true, according to the hypothesized overall theoretical distribution, the probability $p_{i}$ of the value of the $X$ falling into the $i$-th interval $A_{i}$ can be calculated, so $n p_{i}$ is the sample value falling into the $i$-th interval $A_{i}$ The theoretical frequency (theoretical value).

(5) When $\mathrm{H} 0$ is true, the frequency $f_{i} / n$ where the sample value falls in the $i$-th interval $A_{i}$ in $n$ trials should be close to the probability $p_{i}$. When $\mathrm{H} 0$ is not true, the $f_{i} / n$ and $p_{i}$ are very different.

Based on this idea, Pearson introduced the following test statistics:

$$
\chi^{2}=\sum_{i=1}^{k} \frac{\left(f_{i}-n p_{i}\right)^{2}}{n p_{i}}
$$

Under the assumption that the 0 hypothesis holds, it obeys the chi-square distribution with $k-1$ degrees of freedom.

\section{Research result}

\subsection{Variety Distribution of Drug MAH by Approval Time}

As can be seen from Table 1, the years of approval of Chinese drug MAH were mainly concentrated in 2015, with a total of 2720 , accounting for $83.98 \%$. This year was the starting year of the pilot of the national drug marketing license holder system. Year of concentration approved. After 2016, more than 100 new ones are approved each year. In 2014, 41 varieties were also approved. The earliest approval was March 1, 2014. The name of the variety was Zhenyin Ointment. The holder of the marketing authorization was Shandong Boshan Pharmaceutical Co., Ltd. and the production unit was Shandong Health Pharmaceutical Co., Ltd. Chi-square test was performed on the drug marketing license holder varieties and the time distribution of all varieties, and the $\mathrm{P}$ value was 0.000 . This shows that there is a statistical basis for the time difference of varieties under the listing permission holder variety system. 
Table 1. The number of MAH drugs by approval time.

\begin{tabular}{|c|c|c|}
\hline Years & $\begin{array}{c}\text { Number of } \\
\text { Varieties of Drug } \\
\text { MAH }\end{array}$ & $\begin{array}{c}\text { Percentage of all } \\
\text { holder varieties } \\
(\%)\end{array}$ \\
\hline 2014 & 41 & 1.27 \\
\hline 2015 & 2720 & 83.98 \\
\hline 2016 & 158 & 4.88 \\
\hline 2017 & 100 & 3.09 \\
\hline 2018 & 147 & 4.54 \\
\hline 2019 & 73 & 2.25 \\
\hline Total & 3239 & 100.00 \\
\hline
\end{tabular}

Note: The data for 2019 are as of July 31, 2019.

\subsection{Analysis of the types of approved varieties and the corresponding relationship between their holders and production units}

Table 2 shows that of all the types of drug MAH, the number of chemical drugs is the largest (2246) and the largest proportion $(69.24 \%)$, indicating that chemicals are the most important category of drug marketing permission holders. Traditional Chinese medicine accounts for $30.23 \%$ of all varieties, and the number of biological products is the smallest, only 14, accounting for $0.43 \%$. In terms of the corresponding relationship between the listing permission holder and the production unit, a total of 2212 varieties of MAH are consistent with the production unit, accounting for $68.29 \%$, while 1027 varieties are commissioned production, that is, listed The license holder is inconsistent with the production unit. In terms of subdivided product types, in addition to biological products, the proportion of the other two product types (chemical drugs, Chinese medicines) MAH and production units are also close to $68 \%$. A chi-square test was performed on the types of approved drugs and the distribution of all types of drugs, and the $\mathrm{P}$ value was 0.0968 . It shows that the number of MAH varieties and all varieties has a significant positive distribution relationship at a significant level of $10 \%$.

Table 2. The approved type of MAH drugs and the correspondence between the holder and the production unit

\begin{tabular}{|c|c|c|c|c|}
\hline \multirow{2}{*}{$\begin{array}{l}\text { Variety } \\
\text { types }\end{array}$} & \multirow{2}{*}{$\begin{array}{l}\text { Number } \\
\text { of } \\
\text { varieties }\end{array}$} & \multirow{2}{*}{$\begin{array}{c}\text { Proportio } \\
\text { n (\%) }\end{array}$} & \multicolumn{2}{|c|}{$\begin{array}{c}\text { Marketing } \\
\text { Authorization Holder } \\
\text { and Production Unit }\end{array}$} \\
\hline & & & $\begin{array}{l}\text { Consisten } \\
\text { t quantity }\end{array}$ & $\begin{array}{c}\text { Inconsist } \\
\text { ent } \\
\text { quantity }\end{array}$ \\
\hline Chemical & 2246 & 69.34 & 1548 & 698 \\
\hline $\begin{array}{l}\text { Chinese } \\
\text { medicine }\end{array}$ & 979 & 30.23 & 659 & 320 \\
\hline $\begin{array}{c}\text { Biological } \\
\text { product }\end{array}$ & 14 & 0.43 & 5 & 9 \\
\hline Total & 3239 & 100.00 & 2212 & 1027 \\
\hline
\end{tabular}

\subsection{Dosage form analysis of approved varieties}

Figure 1 shows that the categories with the most variety of dosage forms for approved drug MAH are tablets (1045, 32.26\%), injections $(647,19.98 \%)$, capsules $(334$, $10.31 \%)$, and pills. $(333,10.28 \%)$. These 4 types of formulations accounted for $72.83 \%$ of all varieties. The number of APIs and granules is medium, with 287 and 188 , respectively. In addition to the main dosage forms listed in the figure, the number of other dosage forms is 268, accounting for $8.27 \%$. Chi-square test was conducted on the distribution of drug marketing licensee varieties and the dosage forms of all varieties, and the $P$ value was 0.000 , indicating that the existence of the MAH system has statistical basis for the differences in dosage forms. The distribution characteristics of the dosage forms of all varieties can directly and positively affect the distribution characteristics of the variety dosage forms of drug marketing licensee holders.

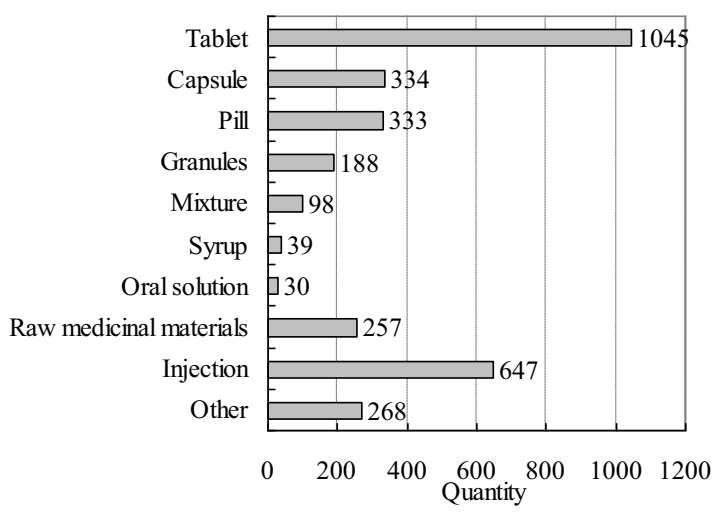

Figure 1. The analysis of MAH drug dosage

\subsection{Geographical Distribution of Drug MAH and Their Varieties}

According to the Decision of the Standing Committee of the National People's Congress on Authorizing the State Council to Carry Out Pilots of Drug MAH in Certain Places and Related Issues, Beijing, Tianjin, Hebei, Shanghai, Jiangsu, Zhejiang, Fujian, Shandong, Guangdong, Sichuan, etc. Ten provinces (municipalities) have launched a pilot system for drug MAH. Therefore, the current geographical distribution of Chinese drug MAH and their varieties is also limited to the above 10 provinces (municipalities). The results in Table 3 show that the number of MAH in Hebei, Guangdong, and Jiangsu ranks in the top three, with 34, 30, and 26 respectively. These three provinces account for $54.55 \%$ of the country's total. Provinces also have the largest number of varieties subject to marketing authorization (Table 4). This shows that Hebei, Guangdong, and Jiangsu have a strong impetus to pilot the system of drug $\mathrm{MAH}$, and applicants are also more active.

There are a total of 197 production units holding varieties of listing authorizations nationwide, which is more than the number of listing authorization holders. In terms of the number of production units, the top three are still Hebei, Guangdong, and Jiangsu, with more than 30 production units. In terms of proportion, the drug varieties produced in these three provinces accounted for $82.8 \%$ of the country's total, indicating that Hebei, Guangdong, and Jiangsu are the nation's major production bases for drug-licensing holding varieties. 
Table 3. The geographical distribution of MAH and MAH drug

\begin{tabular}{|c|c|c|c|c|}
\hline \multirow{2}{*}{ Provinces } & \multicolumn{2}{|c|}{ MAH } & \multicolumn{2}{c|}{ MAH drugs } \\
\cline { 2 - 5 } & Quantity & $\begin{array}{c}\text { Proportion } \\
(\%)\end{array}$ & Quantity & $\begin{array}{c}\text { Proportion } \\
(\%)\end{array}$ \\
\hline Hebei & 34 & 20.61 & 1206 & 37.23 \\
\hline Guangdong & 30 & 18.18 & 827 & 25.53 \\
\hline Jiangsu & 26 & 15.76 & 503 & 15.53 \\
\hline Beijing & 16 & 9.70 & 273 & 8.43 \\
\hline Zhejiang & 16 & 9.70 & 180 & 5.56 \\
\hline Shandong & 14 & 8.48 & 161 & 4.97 \\
\hline Sichuan & 10 & 6.06 & 28 & 0.86 \\
\hline Fujian & 7 & 4.24 & 25 & 0.77 \\
\hline Shanghai & 8 & 4.85 & 13 & 0.40 \\
\hline Tianjin & 4 & 2.42 & 23 & 0.71 \\
\hline
\end{tabular}

Table 4. The geographical distribution of production units and production of medicines

\begin{tabular}{|c|c|c|c|c|}
\hline \multirow{2}{*}{ Provinces } & \multicolumn{2}{|c|}{ Production units } & \multicolumn{2}{c|}{$\begin{array}{c}\text { Production of } \\
\text { medicines }\end{array}$} \\
\cline { 2 - 5 } & Quantity & $\begin{array}{c}\text { Proportion } \\
(\%)\end{array}$ & Quantity & $\begin{array}{c}\text { Proportion } \\
(\%)\end{array}$ \\
\hline Hebei & 46 & 23.47 & 1337 & 41.29 \\
\hline Guangdong & 41 & 20.92 & 837 & 25.85 \\
\hline Jiangsu & 35 & 17.86 & 507 & 15.66 \\
\hline Beijing & 13 & 6.63 & 130 & 4.01 \\
\hline Zhejiang & 20 & 10.20 & 186 & 5.74 \\
\hline Shandong & 16 & 8.16 & 161 & 4.97 \\
\hline Sichuan & 10 & 5.10 & 26 & 0.80 \\
\hline Fujian & 7 & 3.57 & 25 & 0.77 \\
\hline Shanghai & 4 & 2.04 & 6 & 0.19 \\
\hline Tianjin & 4 & 2.04 & 23 & 0.71 \\
\hline
\end{tabular}

\section{Conclusions and discussion}

There are time differences, variety types and geographical differences in the MAH of drugs. This is the application for examination and approval after the full implementation of the MAH of drugs system, and the patent and intellectual property protection in the process of entrusted production technology transfer. Regional drug regulation has implications. The specific conclusions and discussions are as follows.

\subsection{On the relationship between breed approval time and policy implementation time}

The trial of China's drug marketing license holder system started in 2015 . Therefore, the vast majority $(83.98 \%)$ of the drug marketing license holder varieties were approved in 2015. This is because 2015 was the beginning of the year when the MAH system was implemented, and the system is bound to receive attention. It also shows that the drug MAH in the pilot provinces responded to the system in a timely and rapid manner, and also applied for the varieties that may have been approved before. After 2016, the number of declarations has entered a stable period, and there are no more than 200 varieties of new drug MAH each year. This shows that there is a clear correlation between the approval of varieties and the implementation of policies.
It also shows that the introduction of this policy is highly anticipated and concerned.

\subsection{Differences about breed types}

Drug MAH have the highest proportion of chemical products, reaching $69.34 \%$, which is consistent with the trend of the highest proportion of chemical products of all drug types $(62.97 \%)$, which is determined by the proportion characteristics of the drug product itself. In terms of dosage forms, tablets of pharmaceutical MAH accounted for $32.26 \%$, which was higher than other dosage forms. This trend is basically consistent with the trend of the proportion of tablets $(34.37 \%)$ of all drug varieties. In summary, it is clear that the variety types of drug marketing licensee holders are mainly determined by the characteristics of all drug product types, that is, by the "total base" characteristics of all products, not by the MAH system.

\subsection{About geographical differences}

Hebei, Guangdong, and Jiangsu are important bases for China's pharmaceutical industry. There are many drug manufacturers and many varieties of drugs. In addition, these three provinces are listed as pilot provinces for the drug marketing license holder system, which makes these three provinces the top in the country in terms of both the number of drug MAH and the number of varieties held. In the provinces of Tianjin, Shanghai, and Fujian, the number of pharmaceutical companies is relatively small. Therefore, there are fewer pharmaceutical market licensee holders and their varieties. The MAH system is one of the important reasons for the regional differences in the number of $\mathrm{MAH}$ varieties. There are more pharmaceutical enterprise groups in Hebei, Guangdong, and Jiangsu, and there are often more varieties of drug group MAH for enterprise groups, and they have the characteristics of batch declaration.

\subsection{Enlightenment on the status of application after the full implementation of the drug marketing authorization holder system}

The Chinese pharmaceutical MAH system has been fully implemented in December 2019. In the first year after the launch of the comprehensive policy, it is expected that we will usher in a new round of wave of declaration and approval for the variety of drug MAH. It is recommended that the reporting enterprises refer to the reporting and approval status of the 10 pilot provinces. The application subject should better adapt to the MAH system according to the characteristics of different drug varieties and dosage forms, and aim to improve the product quality and the success rate of drug variety declaration for drug marketing licensee holders. From the perspective of regulatory review, for drug manufacturers and provinces with many varieties, it is important to strengthen the deployment of professional reviewers mainly in the areas of chemicals, tablets and injections. Under the MAH system, the supervisory authority should provide 
differentiated and targeted review and management when approving supervision based on the respective characteristics of different drug varieties. It will further strengthen the efficiency of the review and management services to avoid the review lag caused by the concentration of application time, geographical concentration and concentration of drug types, so as to effectively and comprehensively implement the drug marketing license holder system. It is suggested that according to the drug risk situation, the State Drug Administration may decentralize the approval of some low-risk drugs.

\subsection{Enlightenment on patent and intellectual property protection in the process of entrusted production technology transfer}

Drug technology transfer is an important part of a drug's entire life cycle. The difference between the drug MAH system and the current management system (drug approval number and manufacturing company bundling model) is that the MAH system allows holders to entrust a production company to produce drugs, and holders are no longer restricted by their own production qualifications. The pharmaceutical industry can be industrialized by commissioned production as soon as possible, making full use of existing capacity. But at the same time, it also needs to bear the risk of leakage of technology secrets such as patents and intellectual property rights caused by the technology transfer process. In view of this, the holder and the entrusted production enterprise shall strictly perform the signed entrustment agreement and quality agreement. The statistical results of this study can provide decision-making references for actors in the protection of patents and intellectual property rights in the process of technology transfer of commissioned production in various aspects such as MAH varieties, dosage forms, and geographical distribution.

\section{Acknowledgement}

This research was financially supported by the Science and Technology Innovation Project of Guangdong Food and Drug Administration(2018TDZ08).

\section{References}

1. Q.Q.HAN \& Q.ZHANG. The study on implementation and suggestions of drug marketing authorization holder in China. Chinese Journal of New Drugs. 28,5(2019)

2. C.G.Wang. Holder of Drug Marketing Authorization_ A Breakthrough in the Reform of Drug Registration System in China. China Food and Drug Administration. 14,7(2016)

3. Z.S.Yan. Discussion of regulatory strategy for contract manufacturing under marketing authorization holder system. Shanghai Medica. 39,13(2018)
4. Magnolia. Comparison of Drug Marketing Authorization Systems at Home and Abroad. West china journal of pharmacy. 32,3(2017)

5. State Council:Opinions on Reforming the System of Review, Approval and Approval of Drug Therapeutic Devices.(2015)

6. National People's Congress Standing Committee: "Decision of the Standing Committee of the National People's Congress on Authorizing the State Council to Carry Out Pilot Programs of Drug Marketing Authorization Holders in Some Places and Related Issues".(2015)

7. General Office of the State Council: "Notice of the General Office of the State Council on Printing and Distributing Pilot Schemes for the Holder of Drug Marketing Authorization".(2016)

8. State Food and Drug Administration: "Notice of the General Administration on Promoting the Pilot Work of the Drug Marketing Authorization Holder System".(2017)

9. State Council: "Amendment to the Drug Administration Law of the People's Republic of China (Draft for Comment)". (2017) 